\title{
Broad Histogram Relation Is Exact
}

\author{
Paulo Murilo Castro de Oliveira \\ Instituto de Física, Universidade Federal Fluminense \\ av. Litorânea s/n, Boa Viagem, Niterói RJ, Brazil 24210-340 \\ e-mail PMCO @ IF.UFF.BR
}

to be published in Eur. Phys. J. B

\begin{abstract}
The Broad Histogram is a method designed to calculate the energy degeneracy $g(E)$ from microcanonical averages of certain macroscopic quantities $N_{\text {up }}$ and $N_{\text {dn }}$. These particular quantities are defined within the method, and their averages must be measured at constant energy values, i.e. within the microcanonical ensemble. Monte Carlo simulational methods are used in order to perform these measurements. Here, the mathematical relation allowing one to determine $g(E)$ from these averages is shown to be exact for any statistical model, i.e. any energy spectrum, under completely general conditions.

We also comment about some troubles concerning the measurement of the quoted microcanonical averages, when one uses a particular approach, namely the energy random walk dynamics. These troubles appear when movements corresponding to different energy jumps are performed using the same probability, and also when the correlations between successive averaging states are not adequately treated: they have nothing to do with the method itself.
\end{abstract}

PACS: $75.40 . \mathrm{Mg}$ 
Consider a statistical system at thermal equilibrium under constant temperature $T$ (we set the Boltzmann constant to unit). The canonical average reads

$$
<Q>_{T}=\frac{\sum_{S} Q_{S} \exp \left(-E_{S} / T\right)}{\sum_{S} \exp \left(-E_{S} / T\right)}
$$

where both sums run over all states $S$ available for the system, each one corresponding to an energy value $E_{S}$ and another value $Q_{S}$ for the particular quantity $Q$ one is interested in (magnetizatiom, for instance). A very useful way to determine such an average is through computer simulational methods, particularly the so-called importance sampling introduced 45 years ago in a seminal paper [1]. The general idea is to construct a Markovian chain of states available for the system. Along this chain, each new state is obtained by performing some random modification (a movement) at the current state in hands. Instead of all possible states, one uses only the finite sub-set obtained by this Markovian process, in order to calculate an approximation for the true average (1). The exponential terms appearing in (1), i.e. the Boltzmann averaging weights, are automatically taken into account during the construction of the Markovian chain: increasing-energy random movements, i.e. $S \rightarrow S^{\prime}$ where $\Delta E=E_{S^{\prime}}-E_{S}>0$, are accepted only with probability $\exp (-\Delta E / T)$.

The value obtained from this recipe could be a very bad approximation, if the user fails in taking into account some fundamental precautions. For instance, consider an Ising model where $N$ spins can point up or down, corresponding to $\Omega=2^{N}$ possible states. In real implementations one has, say, $N \sim 10^{4}$, and the number $M$ of states along the Markovian chain is always much less than $\Omega>10^{3,000}$. Thus, the tiny sub-set with $M$ averaging states may be a biased sample not representing the huge set with $\Omega$ states. The simplest protocol one can adopt to generate a new state from the current one is the single-spin-flip protocol: one chooses randomly one spin, and flips it or not, according to the energy rules described above. By repeating this single-spin-flip process $M$ times $\left(M \approx N=10^{4}\right.$ ), one gets successive states very similar to each other (at most one different spin among $N$ ), which would be statistically correlated. These correlations will introduce systematic errors. Nobody follows this fool protocol. Indeed, one normally considers a new state along the Markovian chain only after at least $N$ spin-flips were tried, i.e. only after a whole-lattice sweep was performed. Another precaution to be taken into account is to discard a certain number of initial states along the Markovian chain, in order to avoid possible biases caused by any oddness of the very first tossed state. This corresponds to wait for a thermalization transient time.

In order to get the average $<Q>_{T}$ as a function of $T$, one must repeat the computer run again and again, for many different fixed values of $T$. This is a waste of computer 
time, compared to another possible method where the whole temperature spectrum could be sampled in just one computer run. Indeed, still following the same recipes, such a method was shown to be possible [2,3], at least in principle. The idea is to perform an analytic reweighting of equation (1), allowing one to obtain the average $\langle Q\rangle_{T^{\prime}}$ for a new temperature $T^{\prime}$, without performing a new computer run. In order to see how this "magic" would be possible, we need first to introduce the microcanonical average

$$
<Q(E)>=\frac{\sum_{S(E)} Q_{S}}{g(E)},
$$

for the same quantity $Q$. Now, the sum runs over all states $S(E)$ belonging to the same energy level $E$. The energy degeneracy $g(E)$ counts their number. Instead of keeping the system under a constant temperature $T$, i.e. in contact with an equilibrated heat bath, now the condition is more restrictive: the system is completely isolated from the environment, and its energy is kept constant. Each state $S(E)$ within this energy level enters equally weighted into this microcanonical average. Nevertheless, the same kind of fundamental precautions described above must be followed by the user who tries to approximate this average by random sampling some sub-set of the whole $g(E)$ states.

The terms in both sums appearing in (1) can be arranged in groups corresponding to the same energy, and the canonical average reads now

$$
<Q>_{T}=\sum_{E}<Q(E)>P_{T}(E)
$$

where

$$
P_{T}(E)=\frac{g(E) \exp (-E / T)}{\sum_{E^{\prime}} g\left(E^{\prime}\right) \exp \left(-E^{\prime} / T\right)} .
$$

The temperature $T$ appears only in the Boltzmann weights, the degeneracies $g(E)$ being independent of $T$. One can easily express $<Q>_{T^{\prime}}$ in terms of $<Q>_{T}$ through

$$
P_{T^{\prime}}(E)=\frac{P_{T}(E) \exp \left[E\left(\frac{1}{T}-\frac{1}{T^{\prime}}\right)\right]}{\sum_{E^{\prime}} P_{T}\left(E^{\prime}\right) \exp \left[E^{\prime}\left(\frac{1}{T}-\frac{1}{T^{\prime}}\right)\right]} .
$$

Thus, one needs a single computer run at some fixed temperature $T$, measuring the probability distribution $P_{T}(E)$. This measurement can be performed by accumulating the number of visits to each energy level $E$ on a histogram. During the same computer run, one can also accumulate the successive values of $Q_{S}$ in another $E$-histogram, allowing the determination of $\langle Q(E)>$ which also is, of course, independent of $T$. Then, the average 
$<Q>_{T^{\prime}}$ can be obtained by using first equation (5) and then replacing $T$ by $T^{\prime}$ in (3). This is the essence of the so-called reweighting methods [2,3]. Unfortunately, the probability distribution $P_{T}(E)$ is very sharply peaked around the average energy $\langle E\rangle_{T}$, and its numerical evaluation is accurate only inside a tiny region around this peak. Unless $T^{\prime}$ and $T$ are very near to each other, the overlap between $P_{T}(E)$ and $P_{T^{\prime}}(E)$ is negligible, and equation (5) is useless. The new peak position $\langle E\rangle_{T^{\prime}}$ corresponds to the vanishing tail of the actually measured distribution $P_{T}(E)$, where the statistics is poor. Thus, one has no good accuracy at all near the peak of the inferred distribution $P_{T^{\prime}}(E)$. The larger the system size the worse becomes this problem, due to the probability distribution sharpness.

That is why, in spite of the nice reasoning, reweighting methods have difficulties in practice - see, for instance [4]. Nevertheless, the obvious but fundamental observation that both $g(E)$ and $\langle Q(E)>$ do not depend on the particular temperature $T$ adopted in the computer simulation remains an important information. Yet more fundamental is the observation that both $g(E)$ and $\langle Q(E)\rangle$ do not depend on any thermodynamic constraints, that they are characteristics of the energy spectrum alone and not of the particular interactions between the system and its environment. Thus, in principle, it is possible to devise some computer simulational method allowing the direct determination of these quantities. Concerning the degeneracy $g(E)$, many such methods were tried see, for instance [5] — all of them relying on the histogram of visits to each energy level.

The Broad Histogram Method [6] differs from all other methods I know. It relies on the determination of $g(E)$ from the microcanonical averages, i.e. equation (2), of two particular macroscopic quantities also introduced in [6]. First, let's consider some protocol of allowed changes (movements) to be performed at the current state. For instance, one can consider all possible single-spin-flips for the case of an Ising system. Alternatively, one can think about all two-spin-flips, to flip entire blocks containing up to $n$ spins, or any other protocol. Also for other models than Ising's, one can previously determine to adopt any protocol of allowed movements. The only important point to be noted is the microreversibility of such a protocol, i.e. if some movement transforming state $S$ into state $S^{\prime}$ is allowed by the particular protocol, then the back transformation of $S^{\prime}$ into $S$ is also allowed, independent of probabilities. Consider two energy levels $E$ and $E+\Delta E$. Starting from a given state $S$ with energy $E$, the number $N_{\text {up }, S}^{(\Delta E)}$ counts all possible movements increasing its energy by $\Delta E$. One needs to consider all possible $S^{\prime}$ which can be achieved from the fixed current $S$, provided the movement $\left(S\right.$ to $\left.S^{\prime}\right)$ is allowed by the previously adopted protocol, and the energy jump is $\Delta E$. Now, considering all the $g(E)$ states belonging to level $E$, the total number of possible movements increasing the energy from $E$ to $E+\Delta E$ is 


$$
\sum_{S(E)} N_{\mathrm{up}, S}^{(\Delta E)}=g(E)<N_{\mathrm{up}}(E)>,
$$

where the definition (2) of microcanonical average was used. Analogously, starting from some state $S^{\prime}$ with energy $E+\Delta E, N_{\mathrm{dn}, S^{\prime}}^{(\Delta E)}$ is the number of possible movements decreasing its energy to $E$. The total number of possible movements from level $E+\Delta E$ to level $E$ is

$$
\sum_{S^{\prime}(E+\Delta E)} N_{\mathrm{dn}, S^{\prime}}^{(\Delta E)}=g(E+\Delta E)<N_{\mathrm{dn}}(E+\Delta E)>.
$$

Due to the quoted microreversibility, these two numbers are equal, and one has

$$
g(E)<N_{\mathrm{up}}(E)>=g(E+\Delta E)<N_{\mathrm{dn}}(E+\Delta E)>,
$$

which is the fundamental relation introduced in reference [6]. It is now proven to be exact for any statistical model, i.e. any energy spectrum $g(E)$. Note that in both averages $<N_{\text {up }}(E)>$ and $\left\langle N_{\mathrm{dn}}(E)>\right.$ only movements corresponding to energy jumps $\Delta E$ and $-\Delta E$ respectively must be taken into account.

For any system, relation (8) can be used in order to determine $g(E)$ from the microcanonical averages $\left\langle N_{\text {up }}(E)\right\rangle$ and $\left\langle N_{\mathrm{dn}}(E)\right\rangle$ measured as functions of the energy $E$. These measurements (including also $\langle Q(E)>$ ) can be performed by any Monte Carlo approach, the result's accuracy depending exclusively on the quality of this particular approach - not on equation (8) which is exact. Once these two averages are known, one can determine all the spectrum $g(E)$ from the ground state degeneracy $g\left(E_{0}\right)$ supposed to be previously known. In practice, this previous knowledge is not necessary (except for entropy estimates) because $g\left(E_{0}\right)$ cancels out in equation (4). Thus, the Broad Histogram Method consists in measuring the microcanonical averages $\left\langle N_{\text {up }}(E)\right\rangle,\left\langle N_{\mathrm{dn}}(E)\right\rangle$ and $<Q(E)>$ by using any Monte Carlo approach, the results being stored in $E$-histograms. After that, when the computer simulation is already over, equation (8) allows one to determine $g(E)$, and then equations (4) and (3) can be used in order to determine the canonical averages of interest, for any temperature $T$.

Equation (8) can be put into alternative forms. Taking $\Delta E<<E$, one can approximate it by

$$
\frac{\mathrm{d} \ln g(E)}{\mathrm{d} E}=\frac{1}{\Delta E} \ln \frac{<N_{\mathrm{up}}(E)>}{<N_{\mathrm{dn}}(E)>} .
$$

Moreover, by using a dirty mathematical transformation, one can write also 


$$
\frac{\mathrm{d} \ln g(E)}{\mathrm{d} E}=\ln \frac{<\sum_{\Delta E} N_{\mathrm{up}}(E)^{1 / \Delta E}>}{<\sum_{\Delta E} N_{\mathrm{dn}}(E)^{1 / \Delta E}>},
$$

which could be useful in order to average various values of $\Delta E$ simultaneously, saving computer time. Nevertheless, the dirty trick of introducing the exponent $1 / \Delta E$ inside the average brackets could lead to systematic errors which remain to be verified by the user for each case. For the Ising model in two and three dimensions, for instance, it works very well [7].

Hereafter, we will discuss a particular Monte Carlo approach originally adopted $[6,7]$ in order to calculate the microcanonical averages used within the Broad Histogram Method. Canonical simulations under a fixed temperature $T$ cover only a narrow energy window around the average value $\langle E\rangle_{T}$. In order to obtain the microcanonical averages appearing in (8), this is not a good strategy, because one needs to sample a broad energy range. It does not help much to increase the computer time improving the statistics on the exponentially vanishing tails of the distribution $P_{T}(E)$ : its width does not depend on the computer time. One possible solution is to replace such canonical dynamics by a random walk along the energy axis. This idea was implemented [6] by using a simple rejection rule: any increasing-energy tossed movement is performed only with probability $N_{\mathrm{dn}, S}^{(\Delta E)} / N_{\mathrm{up}, S}^{(\Delta E)}$, where $N_{\mathrm{dn}, S}^{(\Delta E)}$ and $N_{\mathrm{up}, S}^{(\Delta E)}$ are measured at the current state $S$, both corresponding to the same energy difference $\Delta E$ of the tossed movement. Following this rule, the range of visited energies will increase proportionally to $\sqrt{t}$, where $t$ is the computer time. Thus, to obtain a broad energy histogram is a simple matter of having enough computer time, within this RW dynamics (for random walk). Actually, I discovered later that RW dynamics was previously introduced in a nice paper [8], considering the much more general problem of optimization in conflicting-interaction systems. Nevertheless, the dynamics introduced in [8] is distinct from that introduced in [6] in a subtle but fundamental detail discussed later.

The RW dynamics solves the problem of obtaining broad histograms. However, there is no free lunch, and this advantage has a price: the correlations appearing between successive states along the Markovian chain are worse to treat than they are within canonical, fixed temperature dynamics, for which correlations can be eliminated simply by waiting some few whole-lattice sweeps before computing a new averaging state. In this case, this simple precaution is enough, because the energy never jumps very far from the average value $<E>_{T}$, thus the successive states are always thermalized (of course, after the initial discarded transient steps). Within the RW dynamics, on the contrary, big energy jumps occur, and just after one of them the current state is not yet thermalized: it carries biases from the recently visited far-away energies, and must be considered as an odd state 
concerning this new energy level. The solution is not simply to wait for more spin flips, because the energy would jump again and again. One possible solution is to thermalize the current state before computing its contribution to the averages. This can be done by performing a few canonical sweeps under the temperature $T(E)$ corresponding to the current energy level. A rough estimate for this value can be measured at the current state itself, through

$$
\frac{1}{T(E)}=\frac{1}{\Delta E} \ln \frac{N_{\mathrm{up}, S}^{(\Delta E)}}{N_{\mathrm{dn}, S}^{(\Delta E)}}
$$

or, again using the already quoted dirty trick,

$$
\frac{1}{T(E)}=\ln \frac{\sum_{\Delta E}\left(N_{\mathrm{up}, S}^{(\Delta E)}\right)^{1 / \Delta E}}{\sum_{\Delta E}\left(N_{\mathrm{dn}, S}^{(\Delta E)}\right)^{1 / \Delta E}} .
$$

Some few such extra thermalization sweeps were adopted in references [6,7], in between two RW sweeps. We observed that one RW sweep followed by one canonical extra sweep is enough to eliminate the correlations within our numerical accuracy.

Introducing extra canonical simulational steps into a method whose main purpose is to eliminate some problems appearing in canonical simulations, however, is not fair. Nevertheless, our main purpose in references [6,7] was to test whether our fundamental equation (8) is valid or not, because we have not yet proven that it is exact at this time. Now it is proven in general. On the other hand, as already quoted, which particular simulational approach would be adopted in order to calculate the microcanonical averages appearing in (8) is a matter of user's choice. I have obtained very accurate results [9] by adopting a microcanonical simulator [10] instead of the RW dynamics, nothing to do with neither canonical simulations nor RW dynamics.

Finally, I show that RW dynamics introduced in [8] is not so bad as claimed in [11], provided the proper corrections were made into its wrong (in thermodynamic grounds) dynamic rule. The rule in [8] is: 1) compute the numbers $N_{\mathrm{up}, S}$ and $N_{\mathrm{dn}, S}$ of possible movements one could perform at the current state $S$, respectively increasing or decreasing its energy; 2) choose one random movement and perform it according to a probability $p_{\text {dn }}$ proportional to $N_{\text {up }, S}$ in case this movement decreases the energy, or $p_{\text {up }}$ proportional to $N_{\mathrm{dn}, S}$ in case the energy would increase; 3) non-varying-energy movements can be performed at will. According to this rule [8], the probability of performing some tossed movement does not depend on the value of the energy jump $\Delta E$. In order to measure thermal averages, this rule is certainly bad, once different energies are treated within the 
same probability. This fundamental concept was ignored in [11], in spite of being already noted many times [12-15] within microcanonical simulations. In [11], of course, wrong averages were found. However, the purpose of reference [8] is not to compute thermal averages, but only to search for cost-minimum states in complex landscapes. For this purpose, thermodynamic concerns do not matter (although can help sometimes), and this dynamics works very well.

The purpose of reference [6] was to compute thermal averages. Namely, the microcanonical averages of $N_{\mathrm{up}, S}^{(\Delta E)}$ and $N_{\mathrm{dn}, S}^{(\Delta E)}$. Note the difference between these quantities and that used in [8] (last paragraph): now, as already mentioned after equation (8), both quantities correspond to a single value of $\Delta E$. Only movements corresponding to the same energy jump $\pm \Delta E$ appear into the microcanonical averages $\left\langle N_{\text {up }}(E)\right\rangle$ and $<N_{\mathrm{dn}}(E+\Delta E)>$. In order to calculate these averages, the rule in [6] is: 1) choose one random movement, and measure its energy jump $\pm \Delta E ; 2)$ compute the numbers $N_{\text {up }, S}^{(\Delta E)}$ and $N_{\mathrm{dn}, S}^{(\Delta E)}$ of possible movements one could perform at the current state $S$, respectively increasing or decreasing its energy by the same amount $\Delta E$ of the tossed movement; 3 ) perform it according to a probability $p_{\mathrm{dn}}$ proportional to $N_{\mathrm{up}, S}^{(\Delta E)}$ in case this movement decreases the energy, or $p_{\text {up }}$ proportional to $N_{\mathrm{dn}, S}^{(\Delta E)}$ in case the energy would increase; 4) non-varying energy movements can be performed at will. Considering the particular case of the square lattice Ising ferromagnet within the further particularity of the singlespin-flip protocol, one has two different possible values for $\Delta E$. One corresponds to spins surrounded by four parallel neighbours $(i=2$, according to the notation adopted in [11]), which must be flipped according to some probability, say $y$. The other corresponds to spins surrounded by three parallel neighbours, with a single one pointing in the opposite sense $(i=1)$, which must be flipped according to probability $x$. The correct RW dynamics [6] corresponds to take $y=x^{2}$, and not $y=x$ as in [11].

The table shows the wrong RW results (WRW) obtained in [11] for $N_{i}$, with $i=$ $-2,-1,1$ and 2 (we use here the same notation), for the same $80 \times 80$ square lattice Ising ferromagnet, compared with the correct canonical averages (CS, also copied from [11]). Now, I included the correct results I got by using the correct RW dynamics (RW) introduced in [6]. I computed only $32 \times 200$ states per energy level around the desired region. This corrected RW rule [6], different from the one used in [11], is good for computing thermal averages, as already tested in [6,7]. Nevertheless, I need to emphasize once again that this corrected RW rule is not supposed to be confused with the Broad Histogram Method, i.e. equation (8), for which any other microcanonical simulator can be applied. The microcanonical averages appearing in equation (8) could even be calculated from another protocol of allowed movements, completely distinct from that used in order to 
count the numbers $N_{\mathrm{up}, S}^{(\Delta E)}$ and $N_{\mathrm{dn}, S}^{(\Delta E)}$ at the current state.

Even within the corrected RW dynamics [6], some precautions in dealing with the correlations may be important. As already quoted, the extra correlations within RW dynamics, as compared to canonical, fixed temperature simulations, are a consequence of the large energy jumps. Thus, an obvious and simple way to avoid these correlations is just to forbid these jumps. This can be done by dividing the energy axis in adjacent small windows, performing RW dynamics inside each window, sequentially. I have done this for the square lattice Ising ferromagnet, and the results for the average energy and specific heat are displayed in the figure. This is a much more crucial test than that presented in [11] for a single energy value (see table), once it depends on the whole function $g(E)$, along the whole energy axis. As one can see, the quality of the results is the same as obtained first in [7] where canonical steps were introduced in between RW steps, and [9], where another completely different simulational dynamics [10] was adopted. All the three cases, however, share the same status: they were obtained by using the exact equation (8), more specifically its approximation (10).

Concluding, the Broad Histogram Method [6], equation (8), was proven to be exact for any statistical model, or any energy spectrum, under completely general conditions. It serves for determining the energy degeneracies $g(E)$ from the microcanonical averages $<N_{\text {up }}(E)>$ and $\left\langle N_{\text {dn }}(E)>\right.$ measured at constant energies $E$. The number $N_{\text {up }}$ counts the possible modifications, or movements one can perform at the current state increasing its energy by an amount $\Delta E$. Analogously, $N_{\mathrm{dn}}$ counts the number of possible movements decreasing its energy by the same amount.

I am indebted to my collaborators Thadeu and Hans, and also to the people from the Metallurgy Engineering School in Volta Redonda, another campus of my university where I give classes during this term, and where the proof of equation (8) came to my mind.

\section{References}

[1] N. Metropolis, A.W. Rosenbluth, M.N. Rosenbluth, A.H. Teller and E. Teller, J. Chem. Phys. 21, 1087 (1953).

[2] Z.W. Salzburg, J.D. Jacobson, W. Fickett and W.W. Wood, J. Chem. Phys. 3065 (1959).

[3] A.M. Ferrenberg and R.W. Swendsen, Phys. Rev. Lett. 61, 2635 (1988).

[4] E.P. Münger and M.A. Novotny, Phys. Rev. B43, 5773 (1991).

[5] J. Lee, Phys. Rev. Lett. 71, 211 (1993); B. Hesselbo and R.B. Stinchcombe, Phys. 
Rev. Lett. 74, 2151 (1995).

[6] P.M.C. de Oliveira, T.J.P. Penna and H.J. Herrmann, Braz. J. of Physics 26, 677 (1996); also in cond-mat 9610041.

[7] P.M.C. de Oliveira, T.J.P. Penna and H.J. Herrmann, Eur. Phys. J. B1, 205 (1998).

[8] B. Berg, Nature, 361, 708 (1993).

[9] P.M.C. de Oliveira, Int. J. Mod. Phys. C9, 497 (1998).

[10] M. Creutz, Phys. Rev. Lett. 50, 1411 (1983).

[11] B. Berg and U.H.E. Hansmann, Eur. Phys. J. B (1998), to appear.

[12] W.M. Lang and D. Stauffer, J. Phys. A20, 5413 (1987).

[13] C. Moukarzel, J. Phys. A22, 4487 (1989).

[14] P.M.C. de Oliveira, T.J.P. Penna, S.M. Moss de Oliveira and R.M. Zorzenon, J. Phys. A24, 219 (1991).

[15] P.M.C. de Oliveira, Computing Boolean Statistical Models, World Scientific, Singapore (1991).

[16] P.D. Beale Phys. Rev. Lett. 76, 78 (1996).

\section{Figure Caption}

Figure 1 Average energy (dots) and specific heat (crosses) for the $32 \times 32$ square lattice Ising ferromagnet. The continuous lines show the exactly known curves [16], the specific heat peak also blown up in the inset. This is a very rough estimate, with only $32 \times 1920$ Monte Carlo sweeps along the whole energy axis, running in less than 6 minutes on a workstation.

\section{Table Caption}

\begin{tabular}{cllll}
$i$ & \multicolumn{1}{c}{-2} & \multicolumn{1}{c}{-1} & \multicolumn{1}{c}{1} & \multicolumn{1}{c}{2} \\
CS & $0.018853(03)$ & $0.072752(04)$ & $0.331070(11)$ & $0.389694(09)$ \\
WRW & $0.034282(26)$ & $0.057936(19)$ & $0.350240(43)$ & $0.388130(55)$ \\
RW & 0.018835 & 0.072766 & 0.331080 & 0.389678
\end{tabular}

Table 1 To be compared with Table 1 of reference [11], where the wrong results (WRW) obtained from the wrong RW dynamics are now corrected by using the correct RW dynamics (RW) introduced in [6]. The error bars are supposed to be similar to that of WRW. Correct canonical simulations (CS) [11] are also included for comparison. 
specific heat

$32 \times 32$

energy
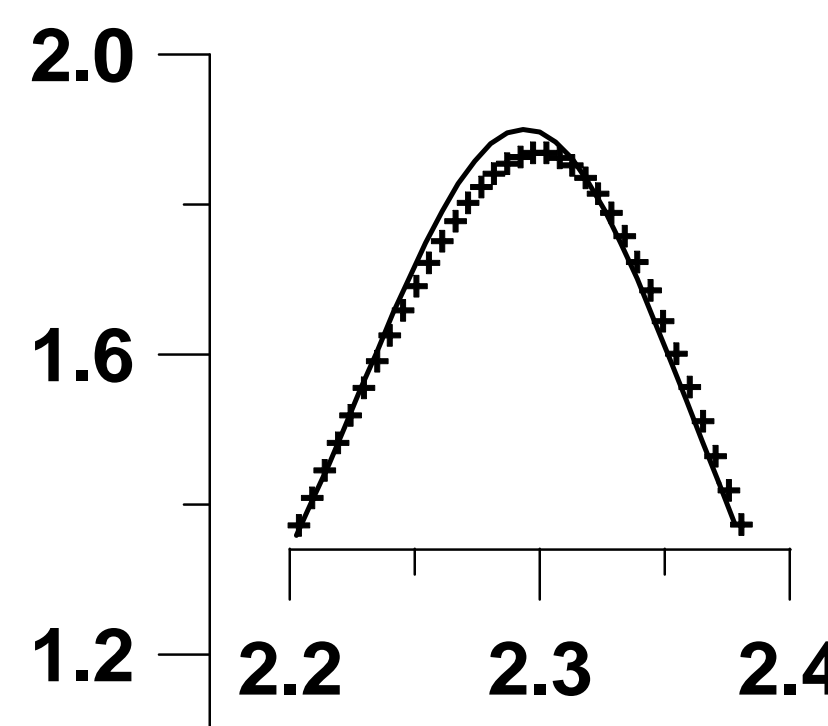

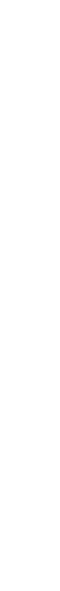

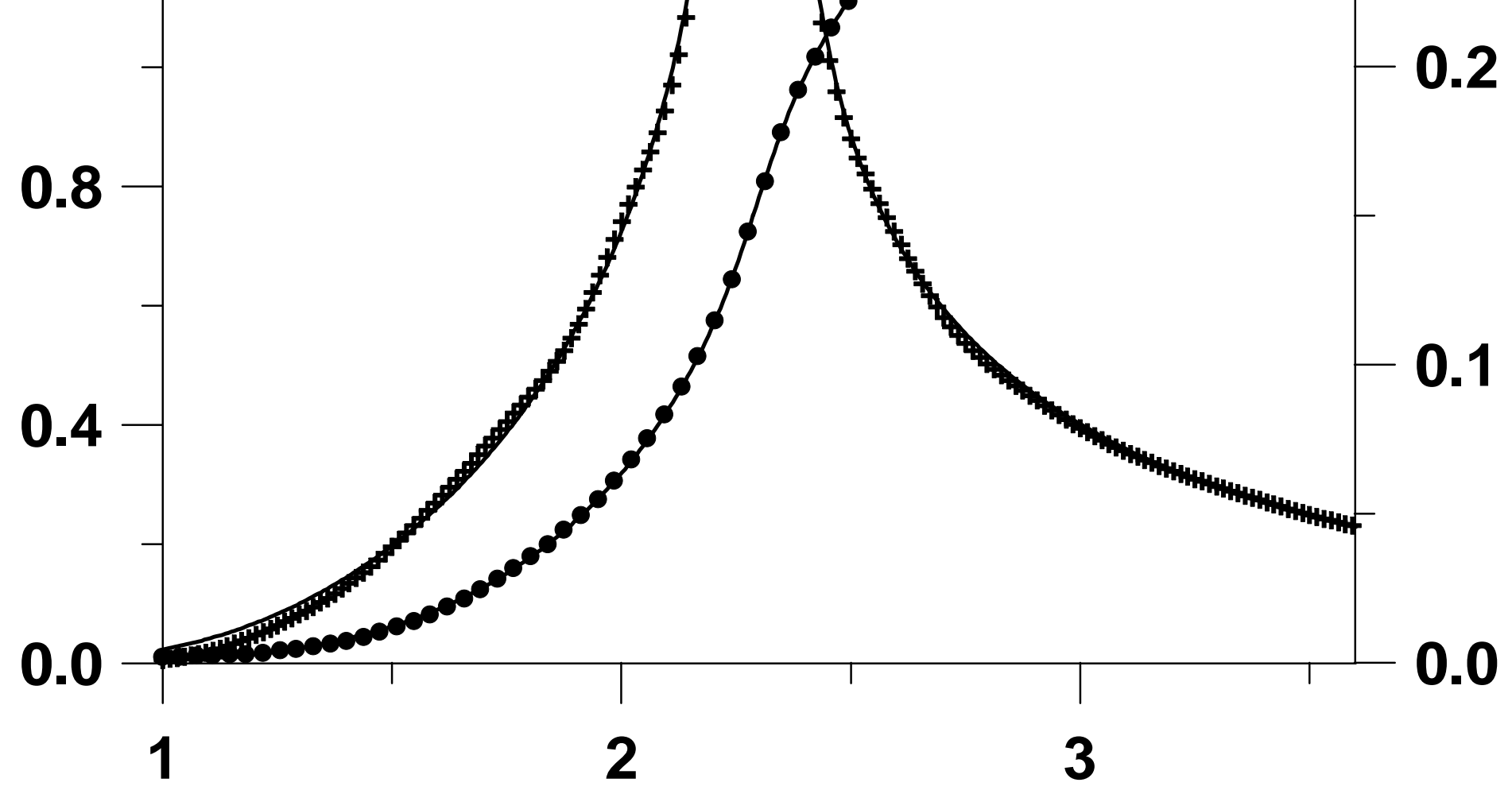

temperature 